\title{
An Elliptic Integral Identity
}

\section{By H. S. Wrigge}

Abstract. The identity

$$
K(\tau)=\frac{1}{(2 \pi)^{1 / 2}} \int_{-\infty}^{\infty} \int_{-\infty}^{\infty} \exp \left[-\frac{1}{2}\left(x^{4}-2\left(2 \tau^{2}-1\right) x^{2} y^{2}+y^{4}\right)\right] d x d y
$$

where $K(\tau)$ is the complete elliptic integral of the first kind, is used to prove that $K(\sqrt{ } 2-1)=\pi^{3 / 2}(2+\sqrt{ } 2)^{1 / 2} / 4 \Gamma\left(\frac{5}{8}\right) \Gamma\left(\frac{7}{8}\right)$.

The identity

(1) $K(\tau)=\frac{1}{(2 \pi)^{1 / 2}} \int_{-\infty}^{+\infty} \int_{-\infty}^{+\infty} \exp \left[-\frac{1}{2}\left(x^{4}-2\left(2 \tau^{2}-1\right) x^{2} y^{2}+y^{4}\right)\right] d x d y,|\tau|<1$, is easily proved by using the standard transformation $x=r \cos v, y=r \sin v$. Before proceeding, we will list a few identities for the benefit of the reader.

$$
\begin{aligned}
& \int_{0}^{\infty} e^{-p t} t^{-1 / 2} e^{-t^{2 / 4 \alpha}} d t=\alpha^{1 / 2} p^{1 / 2} e^{\alpha p^{2} / 2} K_{1 / 4}\left(\frac{1}{2} \alpha p^{2}\right), \quad \operatorname{Re} \alpha>0 \quad \text { [1, p. 146], } \\
& \int_{0}^{\infty} e^{-p t} t^{\mu-1 / 2} K_{\nu+1 / 2}(\alpha t) d t=2^{-1 / 2} \alpha^{-1 / 2} \pi^{1 / 2} \Gamma(\mu-\nu) \Gamma(\mu+\nu+1) s^{-\mu} P_{\nu}^{-\mu}(p / \alpha), \\
& s=\left(p^{2}-\alpha^{2}\right)^{1 / 2}, \quad \operatorname{Re}(\mu+\nu)>-1, \quad \operatorname{Re}(\mu-\nu)>0, \\
& \operatorname{Re} p>-\operatorname{Re} \alpha, \quad[1, \text { p. 198], }
\end{aligned}
$$

$$
P_{p}^{a}(0)=\frac{2^{a} \pi^{1 / 2}}{\Gamma\left(\frac{1}{2}(p-q)+1\right) \Gamma\left(\frac{1}{2}(-p-q+1)\right)} \quad[2, \text { p. 354]. }
$$

We will now calculate the integral (1) in a different way.

Integration with respect to $x$ yields

$$
\begin{aligned}
\int_{-\infty}^{\infty} \exp \left[-\frac{1}{2}\left(x^{4}-2\left(2 \tau^{2}-1\right) x^{2} y^{2}\right)\right] d x=\int_{0}^{\infty} t^{-1 / 2} e^{-t^{2} / 2} e^{\left(2 \tau^{2}-1\right) y^{2} t} d t \\
\quad=\frac{\left(1-2 \tau^{2}\right)^{1 / 2}}{2^{1 / 2}}|y| \exp \left[\frac{\left(2 \tau^{2}-1\right)^{2} y^{4}}{4}\right] K_{1 / 4}\left(\frac{\left(2 \tau^{2}-1\right)^{2} y^{4}}{4}\right), \quad 1-2 \tau^{2}>0 .
\end{aligned}
$$

Integration with respect to $y$ now gives

$$
K(\tau)=\frac{\left(1-2 \tau^{2}\right)^{1 / 2}}{2 \pi^{1 / 2}} \int_{-\infty}^{+\infty}|y| e^{-\nu^{4 / 2}} \exp \left[\frac{\left(2 \tau^{2}-1\right)^{2} y^{4}}{4}\right] K_{1 / 4}\left(\frac{\left(2 \tau^{2}-1\right)^{2} y^{4}}{4}\right) d y .
$$

A few manipulations finally give

Received October 16, 1972.

AMS (MOS) subject classifications (1970). Primary 33A25. 


$$
K(\tau)=\frac{\left(1-2 \tau^{2}\right)^{1 / 2}}{2 \pi^{1 / 2}} \int_{0}^{\infty} t^{-1 / 2} e^{-t\left(1+4 \tau^{2}-4 \tau^{4}\right)} K_{1 / 4}\left(\left(1-2 \tau^{2}\right)^{2} t\right) d t
$$

Using Eq. (3) we can write

$$
K(\tau)=\frac{\pi}{2\left(1-2 \tau^{2}\right)^{1 / 2}} P_{-1 / 4}\left(\frac{1+4 \tau^{2}-4 \tau^{4}}{\left(1-2 \tau^{2}\right)^{2}}\right) .
$$

To get (6) in a more convenient form, we use the well-known formula

$$
K(i \tau)=\frac{1}{\left(1+\tau^{2}\right)^{1 / 2}} K\left(\frac{\tau}{\left(1+\tau^{2}\right)^{1 / 2}}\right) .
$$

Then

$$
K\left(\frac{\tau}{\left(1+\tau^{2}\right)^{1 / 2}}\right)=\frac{\pi\left(1+\tau^{2}\right)^{1 / 2}}{2\left(1+2 \tau^{2}\right)^{1 / 2}} P_{-1 / 4}\left(\frac{1-4 \tau^{2}-4 \tau^{4}}{\left(1+2 \tau^{2}\right)^{2}}\right)
$$

Now put $1-4 \tau^{2}-4 \tau^{4}=0$ which yields $\tau^{2}=-\frac{1}{2}(-) \frac{1}{2} \sqrt{ } 2$. A little calculation and Eq. (4) give

$$
K(\sqrt{ } 2-1)=\frac{\pi^{3 / 2}(2+\sqrt{ } 2)^{1 / 2}}{4 \Gamma\left(\frac{5}{8}\right) \Gamma\left(\frac{7}{8}\right)} \cdot \quad \text { Q.E.D. }
$$

The identity (8) has been proved previously; see for instance [3, pp. 535-536]. The present derivation seems, however, to give a better insight into the problem.

Research Institute of the Swedish National Defense 10450 Stockholm 80,

Sweden

1. A. ERdélyi ET AL., Tables of Integral Transforms. Vol. 1, McGraw-Hill, New York, 1954. MR 15, 868.

2. I. M. Ryžix \& I. S. GRadště̌n, Summen-, Produkt- und Integral-Tafeln, 2nd rev. ed., VEB Deutscher Verlag, Berlin; Plenum Press, New York, 1963. MR 28 \#1326.

3. M. L. GlasseR \& V. E. WOOD, "A closed form evaluation of the elliptic integral," Math. Comp., v. 25, 1971, pp. 535-536. 